\title{
Effects of naloxone and naltrexone on morphine- elicited changes in hamster locomotor activity
}

\author{
PAUL SCHNUR \\ University of Southern Colorado, Pueblo, Colorado
}

\begin{abstract}
Four experiments investigated the effects of naloxone $(0.4 \mathrm{mg} / \mathrm{kg})$ and naltrexone $(1.0 \mathrm{mg} / \mathrm{kg})$ on morphine $(15 \mathrm{mg} / \mathrm{kg})$-elicited sedation and hyperactivity in the hamster. Experiments 1 and 2 demonstrated that naloxone blocks morphine sedation and hyperactivity, respectively. Experiments 3 and 4 demonstrated that naltrexone blocks morphine sedation and hyperactivity, respectively. Moreover, in Experiments 2 and 4, morphine-elicited hyperactivity was replaced by sedation following administration of the antagonists. In Experiment 1, morphine-elicited sedation was replaced by hyperactivity following administration of naloxone. These results are consistent with the hypothesis that morphine's biphasic time-effect curve is the behavioral resultant of two underlying processes.
\end{abstract}

Morphine produces sedative as well as excitatory effects on locomotor activity in the hamster: In comparisons with saline controls, it has been shown that morphine in doses ranging from 0.5 to $40 \mathrm{mg} / \mathrm{kg}$ produces an initial dose-related decrease in activity followed by a dose-related rate of recovery and then a phase of hyperactivity (Schnur, Bravo \& Trujillo, 1983b; Schnur, Bravo, Trujillo, \& Rocha, 1983). With repeated morphine administration, the initial sedation decreases and the subsequent hyperactivity increases (Schnur et al., 1983b; Schnur et al., 1983). Parallel findings have been reported in the rat (Babbini \& Davis, 1972; Vasko \& Domino, 1978), where it has also been observed that naloxone antagonizes morphine-elicited sedation and hyperactivity (Vasko \& Domino, 1978). The purpose of the present work was to test the effects of two opiate antagonists, naloxone and naltrexone, on morphine-elicited changes in hamster locomotor activity. If morphine-elicited sedation and hyperactivity in the hamster are attributable to morphine's interaction with an opiate receptor, then opiate antagonists should block both effects.

\section{EXPERIMENT 1}

The purpose of Experiment 1 was to test the effect of naloxone on morphine-elicited sedation in the hamster. A single naloxone injection $(0.4 \mathrm{mg} / \mathrm{kg})$ was given prior to an injection of morphine $(15 \mathrm{mg} / \mathrm{kg})$. This choice of doses was based upon the following considerations:

This research was supported by a NIH Minority Biomedical Research Support Grant (\#RR-08197-01) to the University of Southern Colorado (Paul Schnur, principal investigator). Naltrexone hydrochloride was donated by Endo Laboratories, Inc. The author is grateful to Peter Barela, Victor Raigoza, and Larry Medina for their assistance with data collection and to C. J. Ksir and P. Kulkosky for their comments on an earlier version of this manuscript.

The author's mailing address is: Center for Psychology and Mental Health, University of Southern Colorado, Pueblo, CO 81001.
(1) Previous work in our laboratory had indicated that a $15-\mathrm{mg} / \mathrm{kg}$ dose of morphine has reliable effects on hamster locomotor activity (Schnur \& Barela, 1984); (2) a 0.4$\mathrm{mg} / \mathrm{kg}$ dose of naloxone has been shown to be an effective opiate antagonist in several response systems (Blumberg \& Dayton, 1972, 1973); and (3) both doses are employed routinely in humans for analgesia $(15 \mathrm{mg} / \mathrm{kg}$ morphine) and for antagonism of narcotic overdose (0.4 $\mathrm{mg} / \mathrm{kg}$ naloxone).

\section{Method}

Subjects. Sixteen adult golden Syrian hamsters (10 females, 6 males), with a mean weight of $95 \mathrm{~g}$, were used. The hamsters were descended from animals obtained from Sasco, Inc. (Omaha, NE), housed individually, maintained on a 12:12 lighting cycle (lights on at 7 a.m.), and given free access to tap water throughout the experiment. The animals received a daily food ration (Purina Rodent Lab (how) after each experimental session, sufficient to maintain $90 \%$ of their ad-lib weights.

Apparatus and Materials. The apparatus consisted of eight identical activity wheels (Wahmann Co., Model LC-34) housed in a room dimly illuminated by two 15 -W bulbs. Running wheels were fitted with microswitches and interfaced (Lafayette minicomputer interface, Model 1180) to an Apple II Plus computer to record the number of wheel revolutions. An ambient noise level of $79 \mathrm{~dB}$ (re: 0.0002 dynes $/ \mathrm{cm}^{2}$, A scale) was maintained.

Morphine injections consisted of $15-\mathrm{mg} / \mathrm{kg}$ doses of morphine sulfate (Lilly), and naloxone injections consisted of $0.4-\mathrm{mg} / \mathrm{kg}$ doses of naloxone hydrochloride (Endo). All injections were administered subcutaneously in the dorsal surface of the neck in $1-\mathrm{ml} / \mathrm{kg}$ volumes.

Procedure. A mixed factorial design was employed, with the contents of a first injection ( $0.9 \%$ saline or naloxone) manipulated within subjects and the contents of a second injection (saline or morphine) manipulated between subjects. The experiment was conducted on 6 successive days. On each of the first 3 days, the animals were given saline injections and placed in the running wheels for a 2-h baseline session. These sessions served to acclimate the animals to the running wheel and to the handling/injection procedures. Baseline data were used also to create two groups whose total activity during baseline sessions was approximately equal. This did not involve matching by subjects. These groups were then randomly assigned to receive a second injection of either morphine or saline on test days. On Day 4, the animals in each group were tested in 
a 2-h running-wheel session following two injections: Group SAL/SAL $(n=4)$ received two saline injections; Group NLX/SAL $(n=4)$ received a naloxone injection followed by a saline injection; Group SAL/MS $(n=4)$ received a saline injection followed by a morphine injection; Group NLX/MS $(n=4)$ received a naloxone injection followed by a morphine injection. Day 5 was another baseline session in which all animals received a single injection of saline before placement in the running wheels for $2 \mathrm{~h}$. On Day 6, the animals were tested as they had been on Day 4, except that the group assignments were reversed between animals in Groups SAL/SAL and NLX/SAL and between animals in Groups SAL/MS and NLX/MS. That is, those animals given saline in the first injection on Day 4 were given naloxone in the first injection on Day 6 and those given naloxone on Day 4 were given saline on Day 6. This reversal of conditions made it possible to test the effects of naloxone in all animals.

Test procedures were as follows: The animals were weighed and given an injection of saline or naloxone. Ten minutes later, they were given an injection of morphine or saline. Following another 10-min interval, they were placed in the running wheels for two hours. The number of wheel revolutions was recorded every twenty minutes.

\section{Results and Discussion}

Figure 1 shows mean running-wheel activity for all groups during the 2-h test-day sessions. The effect of morphine on hamster locomotor activity is evident in the comparison of Group SAL/MS with Group SAL/SAL. Consistent with earlier reports (Schnur, 1985; Schnur \& Barela, 1984; Schnur et al., 1983b; Schnur et al., 1983), morphine in the present experiment produced sedation followed by recovery and then hyperactivity. At the 15$\mathrm{mg} / \mathrm{kg}$ dose used here, hyperactivity began to appear only during the final minutes of the 2-h test session. The present results also indicate that morphine sedation in the ham- ster was completely blocked by a $0.4-\mathrm{mg} / \mathrm{kg}$ dose of naloxone. That is, the sedation evident in Group SAL/MS was absent in Group NLX/MS. In fact, Group NLX/MS maintained a higher level of activity than did Groups SAL/SAL and NLX/SAL throughout the 2-h tests, an effect that will be discussed further below. Naloxone itself had no effect on locomotor activity (cf. Arnsten \& Segal, 1979; Carey, Ross, \& Enns, 1981), Groups NLX/SAL and SAL/SAL maintaining similar activity levels during the test sessions.

These conclusions are corroborated by a 2 (first injection) $\times 2$ (second injection) $\times 6$ (time blocks) mixed factorial analysis of variance (ANOVA), which indicated that there was no effect of the second injection $(F<1)$, but that the effect of the first injection $[F(1,14)=13.34$, $p$ $<.005]$ and the interaction between the first and second injections $[F(1,14)=10.04, p<.01]$ were significant. In addition, the effect of time blocks $[F(5,70)=17.73$, $\mathrm{p}<.001]$, the interaction between the second injection and time blocks $[\mathrm{F}(5,70)=3.36, \mathrm{p}<.01]$, and the interaction between the first injection, the second injection, and time blocks $[\mathrm{F}(5,70)=2.74, \mathrm{p}<.025]$ were all significant. Post hoc comparisons using Fisher's least significant difference test revealed that Group NLX/MS was significantly $(p<.05)$ more active than Group SAL/SAL after $20,60,80,100$, and $120 \mathrm{~min}$ of the test session.

\section{EXPERIMENT 2}

The purpose of Experiment 2 was to determine whether naloxone would block morphine-elicited hyperactivity in the hamster. To ensure a high level of hyperactivity, mor-

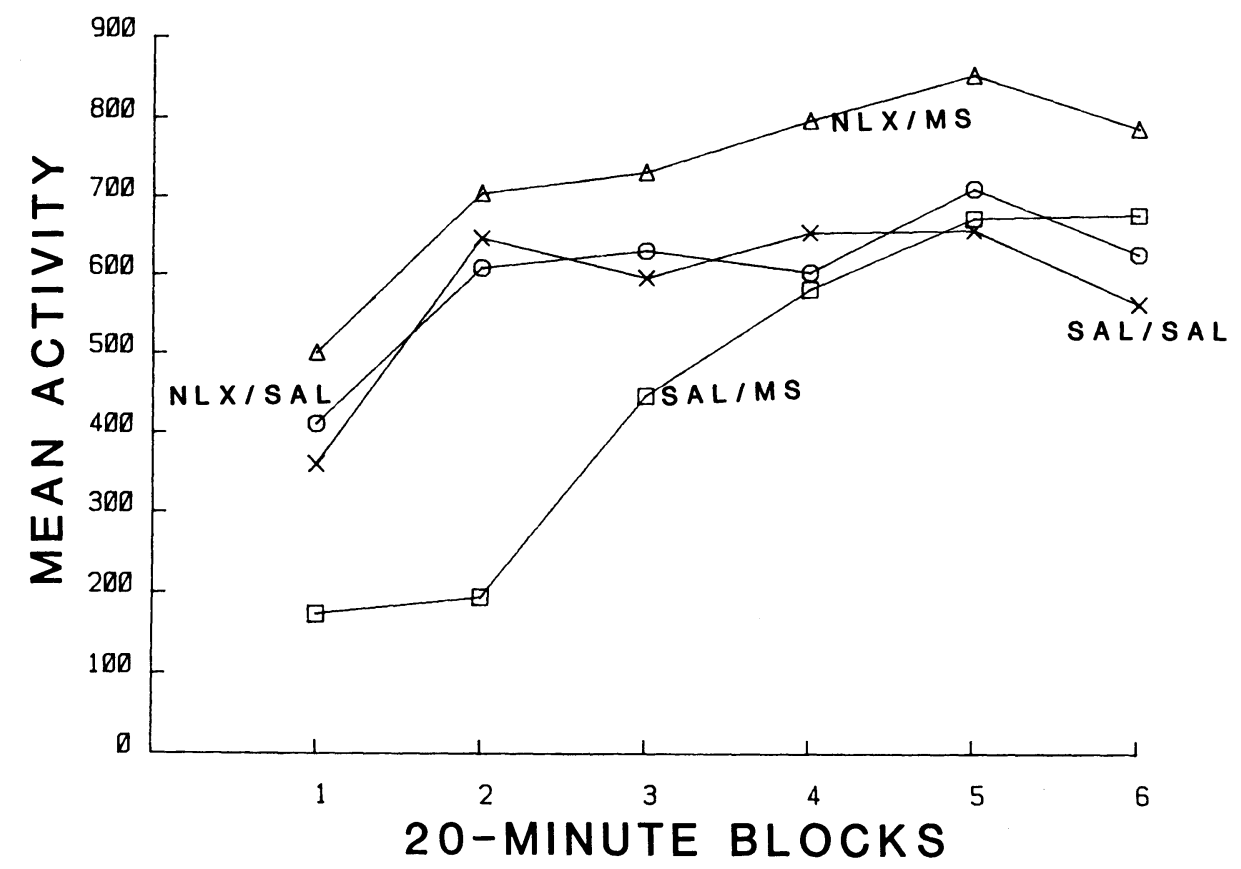

Figure 1. Mean running-wheel activity as a function of 20-min blocks of time shown for all groups $(\mathbf{n}=8)$ in Experiment 1. 
phine $(15 \mathrm{mg} / \mathrm{kg})$ was administered for 3 successive days before the naloxone $(0.4 \mathrm{mg} / \mathrm{kg})$ challenge (Schnur, 1985; Schnur et al. 1983b). Moreover, the naloxone challenge was given $1 \mathrm{~h}$ after animals began running under the influence of morphine. In this way, we expected to synchronize the naloxone injection with the onset of the excitatory portion of morphine's time-effect curve (Schnur, 1985; Schnur, Bravo, \& Trujillo, 1983a).

\section{Method}

Subjects, Apparatus, and Materials. The subjects, apparatus, and materials were identical to those used in Experiment 1.

Procedure. A mixed factorial design was employed, with the contents of a first injection (saline or morphine) manipulated between subjects and the contents of a second injection (saline or naloxone) manipulated within subjects. The animals that had never received morphine in Experiment 1 were assigned to groups that received morphine in the present experiment. Similarly, animals that had received morphine in Experiment 1 were assigned to groups that would not receive morphine (i.e., saline controls) in the present experiment. The experiment commenced 2 days after Experiment 1 was terminated and was conducted on 5 successive days. On each of the first 3 days, half of the animals received a morphine injection $(15 \mathrm{mg} / \mathrm{kg})$ and half received a saline injection before being placed in the running wheels for a 3-h session. On Day 4, the animals were injected twice: Group SAL/SAL $(n=4)$ received two saline injections $1 \mathrm{~h}$ apart; Group SAL/NLX $(n=4)$ received a saline injection followed $1 \mathrm{~h}$ later by a naloxone injection; Group MS/SAL $(n=4)$ received a morphine injection followed $1 \mathrm{~h}$ later by a saline injection; and Group MS/NLX $(n=4)$ received a morphine injection followed $1 \mathrm{~h}$ later by a naloxone injection. On Day 5, the animals were tested as they had been on Day 4, except that group assignments were reversed between the animals in Groups SAL/SAL and SAL/NLX and between the animals in Groups MS/SAL and
MS/NLX. That is, those animals given saline in the second injection on Day 4 were given naloxone in the second injection on Day 5, and those given naloxone on Day 4 were given saline on Day 5 .

The test procedures on Days 4 and 5 were conducted as follows: The animals were weighed and given an injection of saline or morphine. Fifteen minutes later, they were placed in the running wheels for $1 \mathrm{~h}$. They were then removed from the wheels, given an injection of saline or naloxone, and, after a 5-min interval, returned to the running wheels for $2 \mathrm{~h}$.

\section{Results and Discussion}

Figure 2 shows mean running-wheel activity for all groups during the 3-h test sessions. Morphine's biphasic effects on locomotor activity are evident in the comparison of Group MS/SAL with Group SAL/SAL. Compared with Group SAL/SAL, Group MS/SAL was first hypoactive, then hyperactive. The greater hyperactivity in Group MS/SAL of this experiment compared with Group SAL/MS of the first experiment is attributable to the sensitization effects of repeated morphine administration prior to the test sessions of Experiment 2 (Schnur, 1985; Schnur et al., 1983b). The present results indicate that morphineelicited hyperactivity was completely blocked by a $0.4-$ $\mathrm{mg} / \mathrm{kg}$ dose of naloxone. That is, for the first hour after morphine administration, Groups MS/NLX and MS/SAL were equally sedated relative to Groups SAL/NLX and SAL/SAL. After the second injection, however, the performance of Groups MS/NLX and MS/SAL diverged. Following a saline injection, Group MS/SAL was characteristically hyperactive for the remainder of the test session, whereas Group MS/NLX showed no hyperactivity

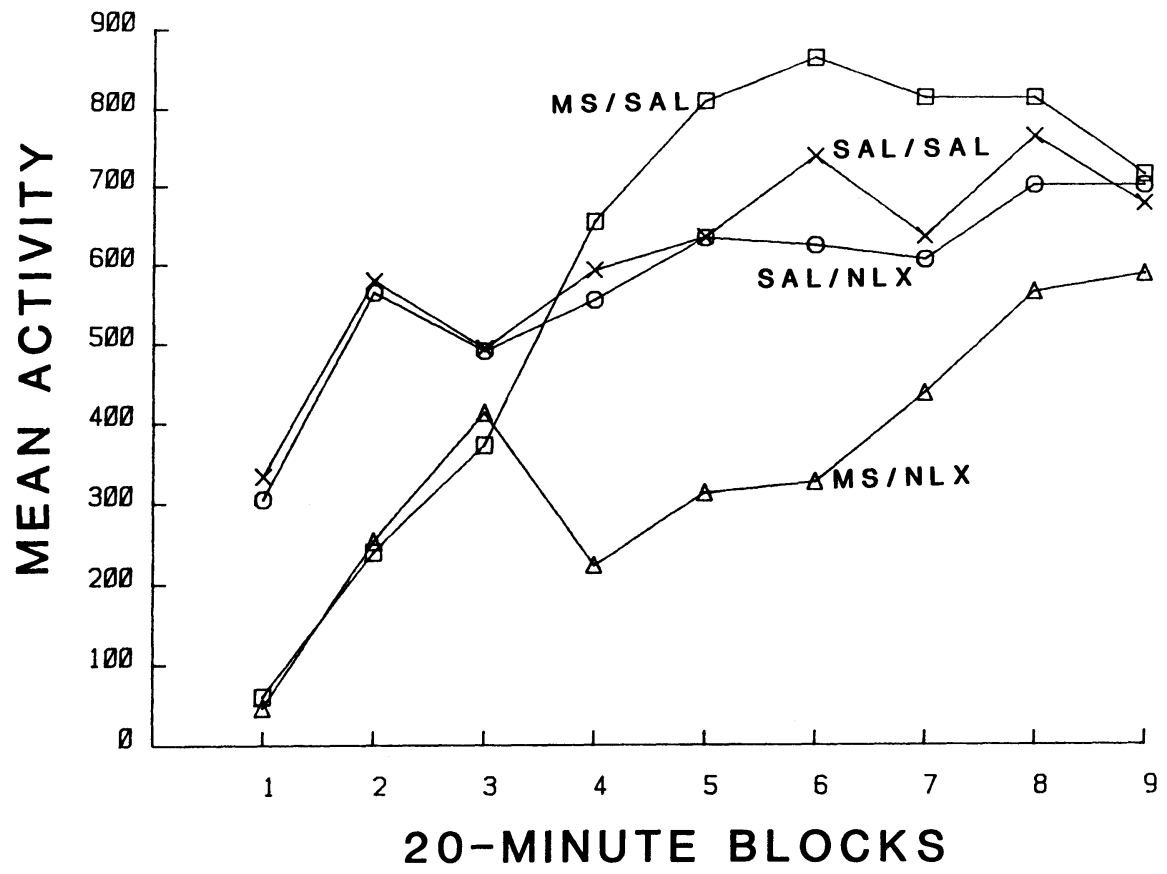

Figure 2. Mean running-wheel activity as a function of 20-min blocks of time shown for all groups $(n=8)$ in Experiment 2. The second injection (saline or naloxone) was given after the third 20-min time block. 
following naloxone. Rather, Group MS/NLX showed a reversal of its recovery from morphine sedation and remained hypoactive relative to saline controls. As in Experiment 1 , naloxone itself had no effect on locomotor activity, Groups SAL/SAL and SAL/NLX maintaining similar activity levels during the test sessions.

These conclusions are corroborated by a 2 (first injection) $\times 2$ (second injection) $\times 9$ (time blocks) mixed factorial ANOVA, which indicated that the effect of the first injection was not significant $(F<1)$, but that the effect of the second injection and the interaction between the effects of the first and second injections were significant $[\mathrm{F}(1,14)=8.29, \mathrm{p}<.01$, and $\mathrm{F}(1,14)=5.07, \mathrm{p}<$ .05 , respectively]. In addition, the effect of time blocks $[F(8,112)=22.06, p<.001]$, the first injection $\times$ time blocks interaction $[\mathrm{F}(8,112)=2.40, \mathrm{p}<.025]$, the second injection $\times$ time blocks interaction $[\mathrm{F}(8,112)=$ $3.25, \mathrm{p}<.005]$, and the first injection $\times$ second injection $\times$ time blocks interaction $[\mathrm{F}(8,112)=2.30, \mathrm{p}<$ .025] were significant.

\section{EXPERIMENT 3}

Experiment 3 was designed to test the effects of naltrexone, an opiate antagonist with longer half-life than naloxone (Blumberg \& Dayton, 1972, 1973; Martin, Jasinski \& Mansky, 1973; Verebey, Volavka, Mule, \& Resnick, 1976), on morphine-elicited sedation and excitation. A single naltrexone injection $(1.0 \mathrm{mg} / \mathrm{kg})$ was given shortly before an injection of morphine $(15 \mathrm{mg} / \mathrm{kg})$. Previous work in our laboratory had indicated that a $1-\mathrm{mg} / \mathrm{kg}$ dose of naltrexone would block morphine-elicited sedation in the hamster (Schnur \& Barela, 1984). Given naltrexone's prolonged duration of action (Blumberg \& Dayton, 1972, 1973; Martin et al., 1973), this single injection was expected to be sufficient to antagonize both portions of morphine's time-effect curve.

\section{Method}

Subjects, Apparatus, and Materials. Sixteen adult golden Syrian hamsters ( 7 males, 9 females), with a mean weight of $104 \mathrm{~g}$ at the beginning of the experiment, were used. Conditions of maintenance and handling were identical to those in Experiment 1. Naltrexone injections consisted of a $1-\mathrm{mg} / \mathrm{kg}$ dose of naltrexone hydrochloride, expressed as the salt, dissolved in $1 \mathrm{ml}$ of $0.9 \%$ saline. In other respects, the apparatus and materials were identical to those used in Experiment 1.

Procedure. The experiment was conducted on 6 successive days. The first 2 days were 3-h saline baseline days, as described above. As in Experiment 1, baseline data were used to create four groups roughly equated for mean activity level. These groups were then assigned randomly to four drug treatment conditions $(n=4)$ : Group SAL/SAL, Group NTX/SAL, Group SAL/MS, and Group NTX/MS. On Days 3-5, Groups SAL/SAL and NTX/SAL received an injection of saline and Groups SAL/MS and NTX/MS received an injection of morphine $15 \mathrm{~min}$ before being placed in the running wheel for a 3-h test session. On Day 6, all animals received two injections, $10 \mathrm{~min}$ apart. The first injection was saline for Groups SAL/SAL and SAL/MS and naltrexone for Groups NTX/SAL and NTX/MS. The second injection was saline for Groups SAL/SAL and NTX/SAL and morphine for Groups
SAL/MS and NTX/MS. Fifteen minutes after the second injection, the animals were placed in the running wheels for a 3-h session. In other respects, the procedure was identical to that of Experiment 1 .

\section{Results and Discussion}

Figure 3 shows mean running-wheel activity during the 3-h test session on Day 6 for all groups. Morphine's biphasic time-effect pattern can be seen in the comparison of Group SAL/MS with Group SAL/SAL. In the comparison of Group SAL/SAL with NTX/SAL, it is evident that a $1-\mathrm{mg} / \mathrm{kg}$ dose of naltrexone had little or no effect on locomotor activity, thus replicating earlier observations (Schnur \& Barela, 1984). It is the comparison of Group NTX/MS with Group SAL/MS that is the focus of this experiment, and it can be seen that although naltrexone antagonized morphine-elicited sedation, there was no evidence that it antagonized morphine-elicited excitation. These data replicate our earlier report of naltrexone antagonism of morphine-elicited sedation in the hamster (Schnur \& Barela, 1984), and they are consistent with the effects of naloxone demonstrated in Experiment 1 above.

Note that the magnitude of naltrexone's effect in the present experiment was smaller than naloxone's effect in Experiment 1. This is somewhat surprising, considering that naltrexone is a more potent opiate antagonist than naloxone in some response systems (Blumberg \& Dayton, 1972, 1973; Martin et al., 1973; Verebey et al., 1976). An explanation of this might be found in the procedure of Experiment 3, which, in contrast to Experiment 1 , involved 3 days of morphine administration before the naltrexone test. Although these days were designed to increase the excitatory portion of morphine's time-effect curve before the naltrexone challenge, at the same time, they probably attenuated morphine's sedative effects, thereby decreasing the sensitivity of the experiment to naltrexone antagonism of morphine sedation (Schnur, 1985; Schnur et al., 1983b).

These conclusions were corroborated by a 2 (first injection) $\times 2$ (second injection) $\times 9$ (time blocks) mixed factorial ANOVA, which revealed that there were no significant differences due to the first injection, the second injection, or their interaction. However, the effects of time blocks was significant $[F(8,96)=14.21, p<.001]$, and the interaction between the second injection and time blocks was marginally significant $[\mathrm{F}(8,96)=1.81,0.10$ $>p>.05]$. A t test indicated that, after $40 \mathrm{~min}$ of the test session, Group SAL/MS was more sedated than Group NTX/MS [t $(6)=1.47,0.10>p>.05]$.

Thus, under the conditions of this experiment, naltrexone produced a short-lived antagonism of morphineelicited sedation, but it failed to antagonize morphineelicited hyperactivity. It is possible that despite the fact that it has a longer half-life than naloxone, naltrexone at the administered dose was not available in sufficient concentration at the time of morphine-elicited excitation. In the next experiment, naltrexone was administered just prior to the onset of morphine-elicited excitation. 


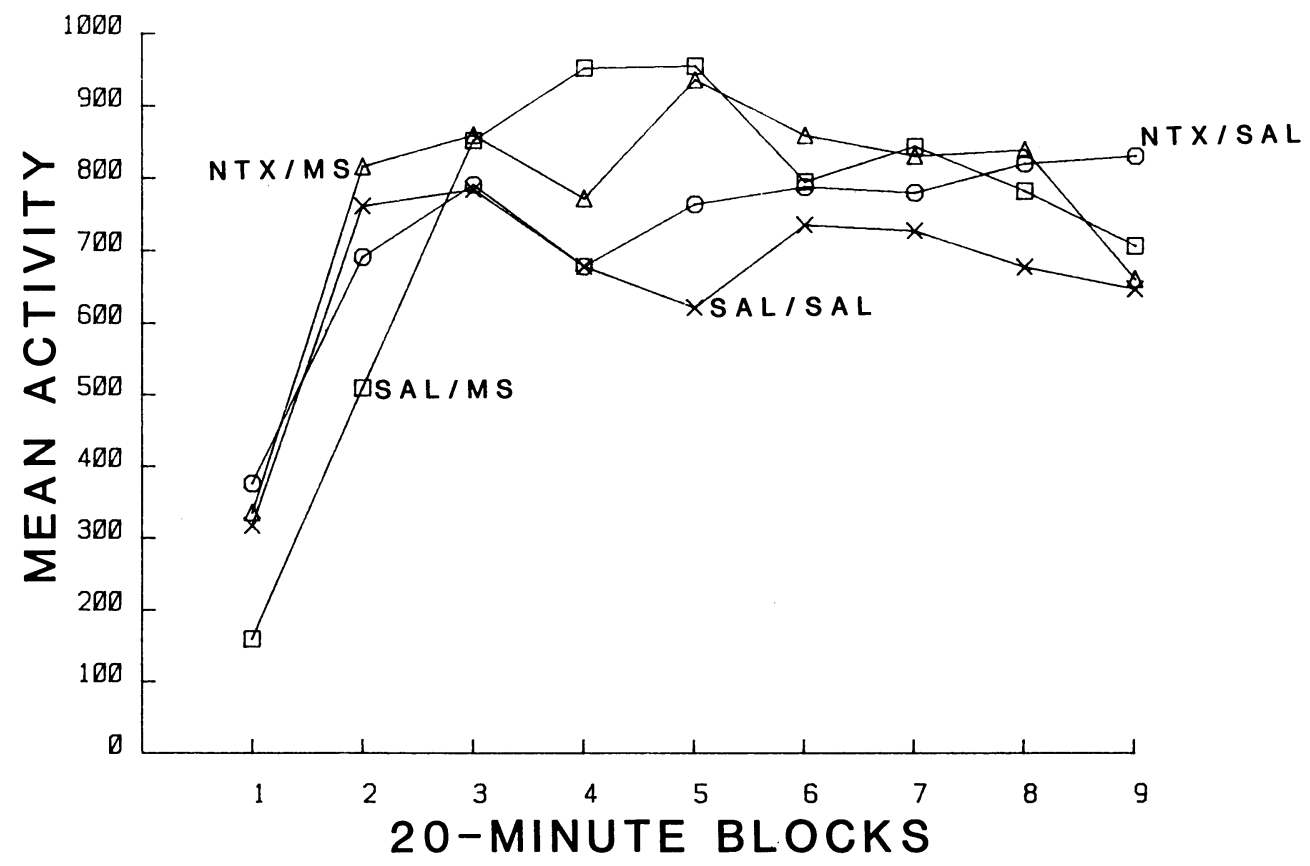

Figure 3. Mean running-wheel activity as a function of 20 -min blocks of time shown for all groups $(n=4)$ in Experiment 3.

\section{EXPERIMENT 4}

This experiment was designed to determine whether naltrexone would block morphine-elicited hyperactivity. Following the procedure of Experiment 2, naltrexone $(1 \mathrm{mg} / \mathrm{kg})$ was given $1 \mathrm{~h}$ after the animals began running under the influence of morphine $(15 \mathrm{mg} / \mathrm{kg})$.

\section{Method}

Subjects, Apparatus, and Materials. Sixteen adult golden Syrian hamsters ( 9 males, 7 females), with a mean weight of $95 \mathrm{~g}$ at the beginning of the experiment, were used. Conditions of maintenance and handling were the same as those in Experiment 3. The apparatus and materials were identical to those of Experiment 3.

Procedure. The experiment was conducted on 5 successive days. The first 2 days were saline baseline days as described above. Four groups were assigned randomly to treatment conditions $(n=4)$. On Day 3, Groups SAL/SAL and SAL/NTX received an injection of saline and Groups MS/SAL and MS/NTX received an injection of morphine $15 \mathrm{~min}$ before a 3-h running-wheel session. The same procedure was followed on Day 4, with the following addition: After $1 \mathrm{~h}$ of running, the animals were removed from the apparatus and given an injection of either saline (Groups SAL/SAL and MS/SAL) or naltrexone (Groups SAL/NTX and MS/NTX). Five minutes later, they were replaced in the running wheels for an additional $2 \mathrm{~h}$. On Day 5, the procedure of Day 4 was repeated, except that group assignments were reversed between the animals in Groups SAL/NTX and SAL/SAL and between the animals in Groups MS/SAL and MS/NTX. That is, those animals given saline in the second injection on Day 4 were given naltrexone in the second injection on Day 5, and those given naltrexone on Day 4 were given saline on Day 5.

\section{Results and Discussion}

Figure 4 shows mean running-wheel activity during the 3-h test sessions for all groups. Recall that the second in- jection (saline or naltrexone) occurred after the third 20min block of the test session. Prior to that time, morphine's sedative effect can be seen in the comparison of Groups MS/SAL and MS/NTX with Groups SAL/NTX and SAL/SAL. It is the comparison of Group MS/SAL with Group MS/NTX, after the first hour, that is the focus of this experiment. Before the second injection, these two groups showed highly similar activity levels. However, following the second injection, the activity of Group MS/SAL continued to increase and exceed that of Group SAL/SAL (i.e., hyperactivity), whereas the activity of Group MS/NTX decreased. Thus, naltrexone completely antagonized morphine-elicited hyperactivity, much as naloxone did in Experiment 2. And, as we found in Experiment 3, naltrexone alone had no effect on hamster locomotor activity.

These conclusions are corroborated by a 2 (first injection) $\times 2$ (second injection) $\times 9$ (time blocks) mixed factorial ANOVA, which revealed that the effect of time blocks $[F(8,112)=7.94, p<.001]$, the interaction between time blocks and the first injection $[F(8,112)=4.17$, $\mathrm{p}<.001]$, the interaction between time blocks and the second injection $[F(8,112)=6.76, p<.001]$, and the interaction between time blocks, the first injection, and the second injection $[F(8,112)=3.80, p<.001]$ were significant. None of the other main effects or interactions was significant.

\section{GENERAL DISCUSSION}

Previously, we reported that morphine produces a biphasic time-effect pattern on hamster locomotor activity 


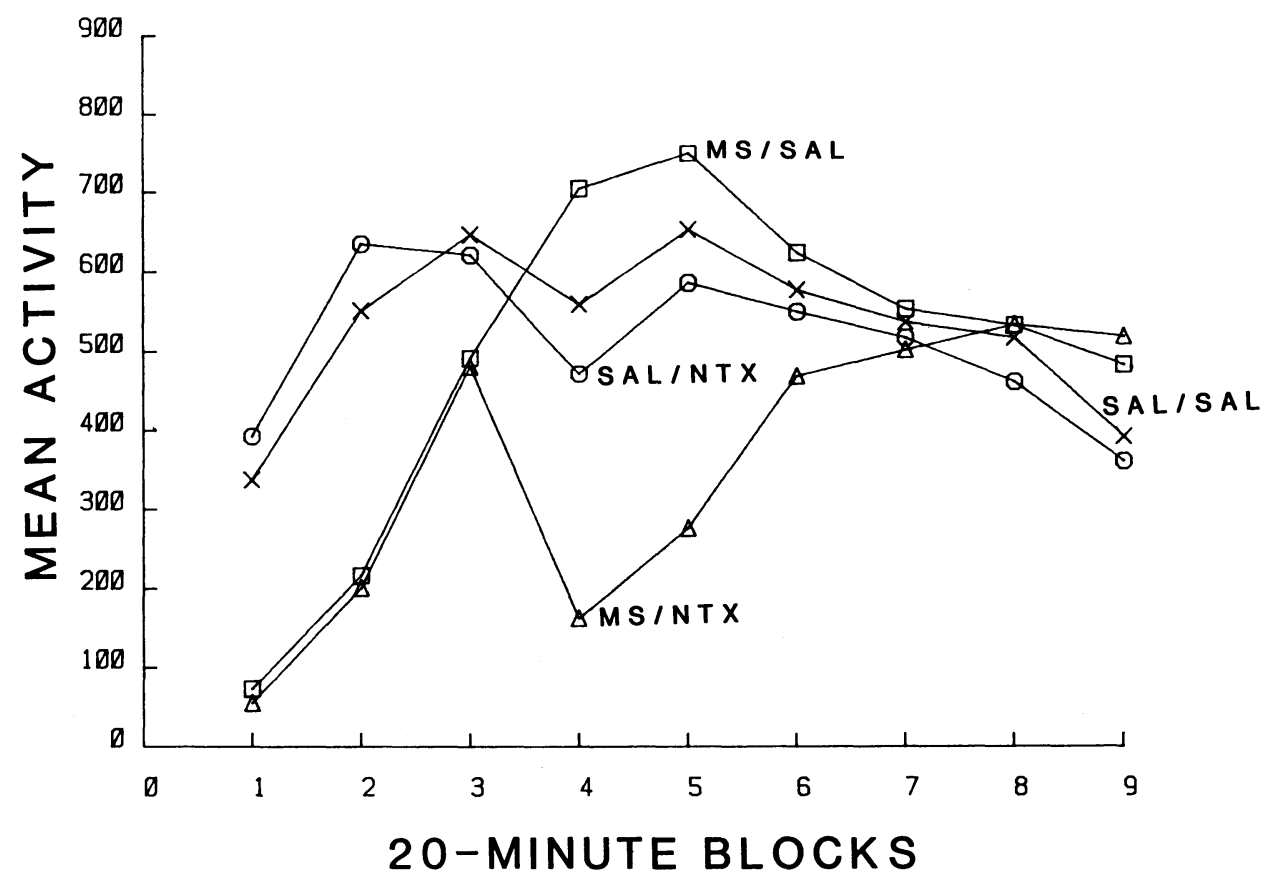

Figure 4. Mean running-wheel activity as a function of 20-min blocks of time shown for all groups $(n=8)$ in Experiment 4. The second injection (saline or naltrexone) was given after the third 20-min time block.

(Schnur et al., 1983) and that, after repeated administration, morphine-elicited hypoactivity decreases whereas morphine-elicited hyperactivity increases (Schnur, 1985; Schnur, et al., 1983). Now, we have adduced evidence that both portions of morphine's time-effect pattern are opiate receptor mediated. Experiments 1 and 3 demonstrated that morphine-elicited sedation is blocked by a prior injection of naloxone or naltrexone; Experiments 2 and 4 demonstrated that morphine-elicited hyperactivity is blocked by a subsequent injection of the same antagonists. These findings have implications for our understanding of morphine's biphasic time-effect pattern and of the changes in that pattern following repeated drug administration.

The present results suggest that morphine's biphasic time-effect pattern is the resultant of two underlying mechanisms, one excitatory, the other inhibitory. That is, following the naloxone injection in Experiment 2, Group MS/NLX showed an immediate decrease in its activity-the hyperactivity seen in Group MS/SAL was converted to sedation in Group MS/NLX. Similarly, following the naltrexone injection in Experiment 4, Group MS/NTX reverted to hypoactivity whereas Group MS/SAL became hyperactive. In both experiments, therefore, opiate antagonists did not merely block the hyperactivity, but, additionally, elicited sedation. That this effect was not engendered by the antagonists themselves can be seen from the performance of Groups SAL/NTX and NLX/SAL, which did not differ from that of saline controls. These results can be explained parsimoniously by assuming that naloxone and naltrexone uncover an inhibi- tory process that is otherwise obscured by hyperactivity. In blocking the hyperactivity, the opiate antagonists reveal the complementary process.

Similarly, the presence of an underlying excitatory process during morphine-elicited sedation is suggested by the results of Experiment 1 . In this case, Group NLX/MS was not only nonsedated, but was more active than the other groups throughout the test session. The sedation evident in Group SAL/MS was converted to hyperactivity by the prior naloxone injection. That this was not a stimulant effect of naloxone itself is attested to by the performance of Groups NLX/SAL and SAL/SAL. Rather, in blocking morphine-elicited sedation, naloxone uncovered an excitatory process that is otherwise masked by sedation.

It might be noted that, in blocking sedation (Experiments 1 and 3), the antagonists were administered prior to a morphine injection, whereas, in blocking hyperactivity (Experiments 2 and 4), the antagonists were administered after a morphine injection. Although the order of administration of the agonist-antagonist pairs thus was confounded with the timing of the injections, it seems unlikely that this factor could account for the present results. Moreover, we have demonstrated elsewhere that, for a range of morphine doses, naloxone blocks morphineelicited sedation whether given just before or just after the morphine injection ( Schnur et al., 1983a).

Thus, we are proposing that morphine elicits two concurrent, but opposing, processes in the hamster. Shortly after an injection, the inhibitory process predominates over the excitatory process and sedation is the behavioral 
resultant. Subsequently, the excitatory process predominates over the inhibitory process and hyperactivity is the behavioral resultant. That the complementary processes exist concurrently is demonstrated by the results of the present experiments: When one process was blocked by an antagonist, its complement became evident. If this interpretation is valid (Babbini \& Davis, 1972; Vasko \& Domino, 1978), then changes in morphine's biphasic time-effect pattern can be accounted for in several ways. For example, the inhibitory process might decrease with repeated drug administration (i.e., tolerance) but the excitatory process remain unchanged. This would lead to a decrease in hypoactivity and an increase in hyperactivity. Or the excitatory process might increase with repeated drug administration (i.e., sensitization) but the inhibitory process remain unchanged. This, too, would lead to a decrease in hypoactivity and an increase in hyperactivity. A third possibility is that both processes change with repeated drug exposure. Additional research will be required to distinguish among these alternatives.

The dual-process interpretation of the present experiments raises an important question about the mechanism through which opiate antagonists influence morphineelicited behaviors. The results of Experiments 1 and 3 provide clear evidence that opiate antagonists block morphine-elicited sedation, and the results of Experiments 2 and 4 provide clear evidence that opiate antagonists block morphine-elicited hyperactivity. According to the dual-process interpretation offered above, both excitation and inhibition are elicited concurrently by morphine. How, then, do the antagonists act selectively? For example, in Experiment 2, naloxone antagonized morphineelicited hyperactivity and Group MS/NLX became sedated; why was this sedation not antagonized by naloxone? Similarly, in Experiment 1, naloxone antagonized morphine-elicited sedation and Group NLX/MS became hyperactive; why was this hyperactivity not antagonized by naloxone? In the context of the dual-process hypothesis, it appears that opiate antagonists act to oppose the predominant process - in other words, naloxone and naltrexone selectively antagonize morphine-elicited behavior.

\section{REFERENCES}

Arnsten, A. T., \& Segal, D. S. (1979). Naloxone alters locomotion and interaction with environmental stimuli. Life Sciences, 25, 1035-1042.

BabBinI, M., \& Davis, W. M. (1972). Time-dose relationships for locomotor activity effects of morphine after acute or repeated treatment. British Journal of Pharmacology, 46, 213-224.

Blumberg, H., \& DAYTON, H. B. (1972). Naloxone and related compounds. In H. W. Kosterlitz, H. O. J. Collier, \& J. E. Villarreal (Eds.), Agonist and antagonist actions of narcotic analgesic drugs. London: Macmillan.

Blumberg, H., \& Dayton, H. B. (1973). Naloxone, naltrexone and related noroxymorphones. In M. C. Braude, L. S. Harris, E. L. May, J. P. Smith, \& J. E. Villarreal (Eds.), Advances in biochemical psychopharmacology: Vol. 8. Narcotic antagonists. New York: Raven.

CAREY, M. P., Ross, J. A. \& EnNs, M. P. (1981). Naloxone suppresses feeding and drinking but not wheel running in rats. Pharmacology Biochemistry \& Behavior, 14, 569-571.

MARTIN, W. R., JASINSKI, D. R., \& MANSKY, P. A. (1973). Naltrexone, an antagonist for the treatment of heroin dependence. Archives of General Psychiatry, 28, 784-791.

SCHNUR, P. (1985). Morphine tolerance and sensitization in the hamster. Pharmacology Biochemistry \& Behavior, 22, 157-158.

SCHNUR, P., \& BARELA, P. (1984). Locomotor activity and opiate effects in male and female hamsters. Pharmacology Biochemistry \& Behavior, 21, 369-374.

Schnur, P., Bravo, F., \& Trujillo, M. (1983a, April). Opiate induced changes in hamster activity. Paper presented at the meeting of the Western Psychological Association, San Francisco.

Schnur, P., Bravo, F., \& Trujillo, M. (1983b). Tolerance and sensitization to the biphasic effects of low doses of morphine in the hamster. Pharmacology Biochemistry \& Behavior, 19, 435-439.

Schnur, P., Bravo, F., Trujlllo, M., \& Rocha, S. (1983). Biphasic effects of morphine on locomotor activity in hamsters. Pharmacology Biochemistry \& Behavior, 18, 357-361.

VASKo, M. R., \& Domino, E. F. (1978). Tolerance development to the biphasic effects of morphine on locomotor activity and brain acetylcholine in the rat. Journal of Pharmacology \& Experimental Therapeutics, 207, 848-858.

Verebey, K., Volavka, J., Mule, S. J., \& Resnick, R. B. (1976). Naltrexone: Disposition, metabolism, and effects after acute and chronic dosing. Clinical Pharmacology \& Therapeutics, 20, 315-328. 